\title{
Pilot Study on Electrocardiogram Derived Respiratory Rate Using a Wearable Armband
}

\author{
Jesús Lázaro ${ }^{1,2,3}$, Raquel Bailón ${ }^{2,3}$, Eduardo Gil ${ }^{2,3}$, Yeonsik Noh $^{4}$, Pablo Laguna ${ }^{2,3}$, Ki H Chon ${ }^{1}$ \\ ${ }^{1}$ Department of Biomedical Engineering, University of Connecticut, Storrs CT, USA \\ ${ }^{2}$ BSICoS Group, Aragón Institute for Engineering Research (I3A), IIS Aragón, University of \\ Zaragoza, Zaragoza, Spain \\ ${ }^{3}$ CIBER in Bioengineering, Biomaterials and Nanomedicine (CIBER-BBN), Madrid, Spain \\ ${ }^{4}$ College of Nursing and Department of Electrical and Computer Engineering, University of \\ Massachusetts, Amherst, MA, USA
}

\begin{abstract}
A pilot study on deriving respiratory rate from electrocardiogram (ECG) signals recorded by a self-developed wearable armband is presented. The armband includes a pair of dry electrodes which record ECG and it is designed for long-term monitoring. Armband-ECG and plethysmography-respiration signals were simultaneously recorded from 5 subjects ( 3 male) while paced breathing at constant rates from 0.1 to $0.4 \mathrm{~Hz}$ (with an increment of 0.1 $\mathrm{Hz}$ ). Respiratory rate was estimated from the armbandECG by using a method based on the variations in QRS slopes and $R$-wave angle. The estimations were compared to those obtained from the respiration signal. Obtained median and interquartile ranges of the relative error were lower than $4 \%$ for every requested respiratory rate. This suggests that normal ranges of spontaneous respiratory rate could be estimated from the wearable armband, allowing us to consider it for long-term wearable cardio and/or respiratory monitoring.
\end{abstract}

\section{Introduction}

Long-term daily monitoring respiration is desirable in a wide range of applications especially when combined with ECG monitoring. These applications include sleep studies [1], prediction of epileptic seizures [2], stress assessment [3], and monitoring of chronic respiratory patients [4].

The common techniques for respiratory monitoring usually require cumbersome devices which are not convenient for daily monitoring, such as chest bands or nasal cannula. However, some alternatives have been presented in the literature based on obtaining respiratory information indirectly from other biomedical signals. Some of them are based on electrocardiogram (ECG), leading to the socalled ECG-derived-respiration (EDR) methods. These methods exploit different respiration-related modulations in beat occurrence and/or in ECG morphology. Beatoccurrence-based methods are known to have problems with non-related low-frequency (below $0.15 \mathrm{~Hz}$ ) modulations which also can be observed in heart rate, while ECG morphology methods are not affected by this phenomenon.

However, the conventional setup which is usually used in the reported EDR studies is based on adhesive wet electrodes over the chest, and this is not convenient for longterm daily monitoring. The use of adhesive wet electrodes cause skin irritation after few (2-3) days limiting their application periods. Furthermore, placing the electrodes over the chest leads to discomfort. In this study, a setup based on a pair of dry electrodes placed over the arm was used. These electrodes were developed in our lab at University of Connecticut [5], as well as an armband incorporating them which can record ECG signals standalone.

This armband device is more convenient for long-term daily monitoring, but it is more challenging than the conventional setup based on wet electrodes over the chest. Typically, the use of dry electrodes leads to a lower signalto-noise ratio than the use of wet electrodes. Moreover, one of the main sources of respiratory modulation in the ECG morphology relies on the movement of the electrodes with respect to the heart, and this movement is reduced when the electrodes are placed over the arm. However, the effect of the impedance changes in the thorax due to the airflow exchange is present, thus, obtaining respiratory information from ECG morphology can be possible using the armband. These differences can affect the morphology of the ECG, and thus the accuracy of EDR methods.

This paper presents a pilot study on how the EDR method presented in [6] performs with ECG signals recorded by the wearable armband. The method presented in [6] is based on QRS-morphology variations, in particular, on QRS slopes and R-wave angles. This choice was 
based on that it can be applied with single-lead ECG signals, and on that it is the best suited for stress test (which remains a highly noisy and non-stationary environment) to the best of our knowledge.

\section{Methods}

\subsection{Data and signal preprocessing}

ECG and respiration signals were simultaneously recorded from 5 subjects ( 3 male) during a paced breathing experiment. Subjects were requested to breath at specific fixed rates by using a visual guidance. Requested rates were $0.1,0.2,0.3$, and $0.4 \mathrm{~Hz}$, covering the range of normal spontaneous breathing rate. Each rate was requested for 2 minutes. Respiration was recorded by using Respibands (Ambulatory Monitoring, Inc., Ardsley NY, USA) which are based on plethysmography. Its digitalization was performed by using a 16-bit A/D converter (PowerLab/4SP, ADInstruments, Inc., Dunedin, New Zealand) at a sampling rate of $100 \mathrm{~Hz}$. ECG was recorded by using the wearable armband developed in our lab at University of Connecticut, at a sampling rate of $1000 \mathrm{~Hz}$. The armband is worn on the left arm as shows Figure 1a.

The ECG preprocessing was similar to that used in [6]. First, a low-pass filter with a cutoff frequency of $35 \mathrm{~Hz}$ was applied. Then, QRS complexes were detected and delineated by the wavelet-based technique described in [7], which offers $n_{\mathrm{Q}_{i}}, n_{\mathrm{R}_{i}}$, and $n_{\mathrm{S}_{i}}$ denoting $\mathrm{Q}, \mathrm{R}$, and $\mathrm{S}$ wave peaks in this paper. In case no $\mathrm{Q}$ wave peak was detected for the $i$-th, QRS onset was used as $n_{\mathrm{Q}_{i}}$. Similarly, QRS offset was used as $n_{\mathrm{S}_{i}}$ if no $\mathrm{S}$ wave peak is detected for the $i$-th beat. Sinus beats were determined by the method presented in [8], and baseline wander was removed by cubic spline interpolation.

\subsection{ECG derived respiration signals}

Three features were computed from each QRS complex: upwards to the R-wave slope $\left(\mathcal{I}_{\mathrm{Us}_{i}}\right)$, downwards from the R-wave slope $\left(\mathcal{I}_{\mathrm{DS}_{i}}\right)$, and R-wave angle $\left(\phi_{i}\right)$.

In order to measure $\mathcal{I}_{\mathrm{Us} i}$, the location of the maximum absolute value of the first derivative of the ECG signal between $n_{\mathrm{Q}_{i}}$ and $n_{\mathrm{R}_{i}}$ was detected. Then, a straight line was fitted to the ECG signal within a window of $8 \mathrm{~ms}$ centered at the location of this maximum. The value of $\mathcal{I}_{\mathrm{Us}_{i}}$ was set as the slope of this straight line. $\mathcal{I}_{\mathrm{DS}_{i}}$ was measured using a similar procedure, this time using the ECG signal between $n_{\mathrm{R}_{i}}$ and $n_{\mathrm{S}_{i}}$. Subsequently, $\phi_{i}$ was measured as the smallest angle formed by the lines fitted for measuring $\mathcal{I}_{\mathrm{Us}_{i}}$ and $\mathcal{I}_{\mathrm{DS}_{i}}$. Figure $1 \mathrm{~b}$ illustrates a descriptive example of how $\mathcal{I}_{\mathrm{US}_{i}}, \mathcal{I}_{\mathrm{DS}_{i}}$, and $\phi_{i}$ are obtained.

These three parameter series are measured beat-to-beat, so they are not evenly sampled. For each one of them,

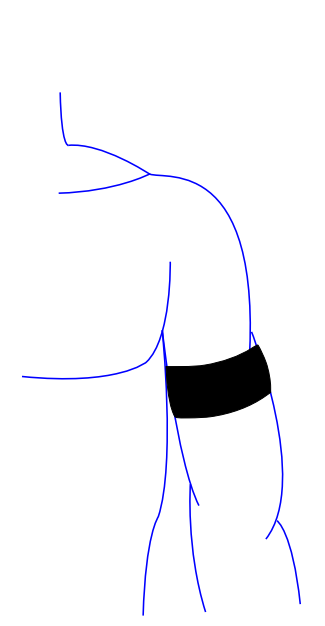

(a)

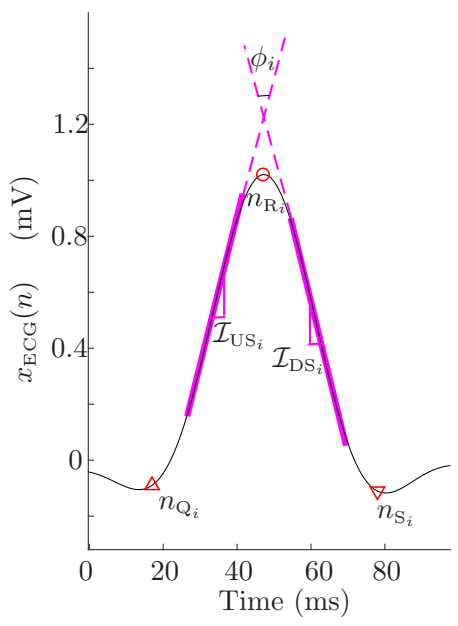

(b)
Figure 1: Armband location (a), and relevant points in the QRS slopes and R-wave angle measurement algorithm (b).

a median-absolute-deviation-based outlier-rejection rule was applied, and a $4-\mathrm{Hz}$ evenly sampled version was obtained by cubic spline interpolation. The resultant parameter series from $\mathcal{I}_{\mathrm{US}_{i}}, \mathcal{I}_{\mathrm{Ds}}$, and $\phi_{i}$ are denoted $d_{\mathrm{US}}(n), d_{\mathrm{DS}}(n)$, and $d_{\mathrm{R}}(n)$, respectively, and they are used as EDR signals in this work. An example of these EDR signals is shown in Figure 2. Further details are given in [6].

\subsection{Respiratory rate measurement}

Respiratory rate was estimated by using the algorithm presented in [6]. It allows to estimate respiratory rate from only one EDR signal, or from combination of several EDR signals to yield a more robust estimation. The algorithm can be divided into 2 stages: the power spectrum density (PSD) estimation, and the "peak-conditioned" average.

The PSD estimation was performed from segments of $42 \mathrm{~s}$, by using the Welch periodogram with subsegments of $12 \mathrm{~s}$ and an overlap of 50\%. The PSD estimated from the $j^{\text {th }}$ EDR signal and the $k^{t h}$ segment of $42 \mathrm{~s}$ is denoted $S_{j, k}(f)$ in this paper. A $S_{j, k}(f)$ is estimated each $5 \mathrm{~s}$.

The "peak-conditioned" average consists of a moving average of only those $S_{j, k}(f)$ which have a "peaky" shape. At each $k$ instant, up to 5 spectra per used EDR signal can be averaged. In order to determine whether $S_{j, k}(f)$ has a peaky shape or not, a respiration peak was chosen based on both amplitude and proximity to a respiratory rate reference $f_{\mathrm{R}}(k-1)$ obtained from previous $(k-1)$ segments. Then, the peakness of $S_{j, k}(f)$ is computed as the percentage of power around the respiratory peak with respect to the total power in a wider band around $f_{\mathrm{R}}(k-1)$ where respiration is expected to be.

Subsequently, the "peak-conditioned" average $\bar{S}_{\mathrm{k}}(f)$ is 

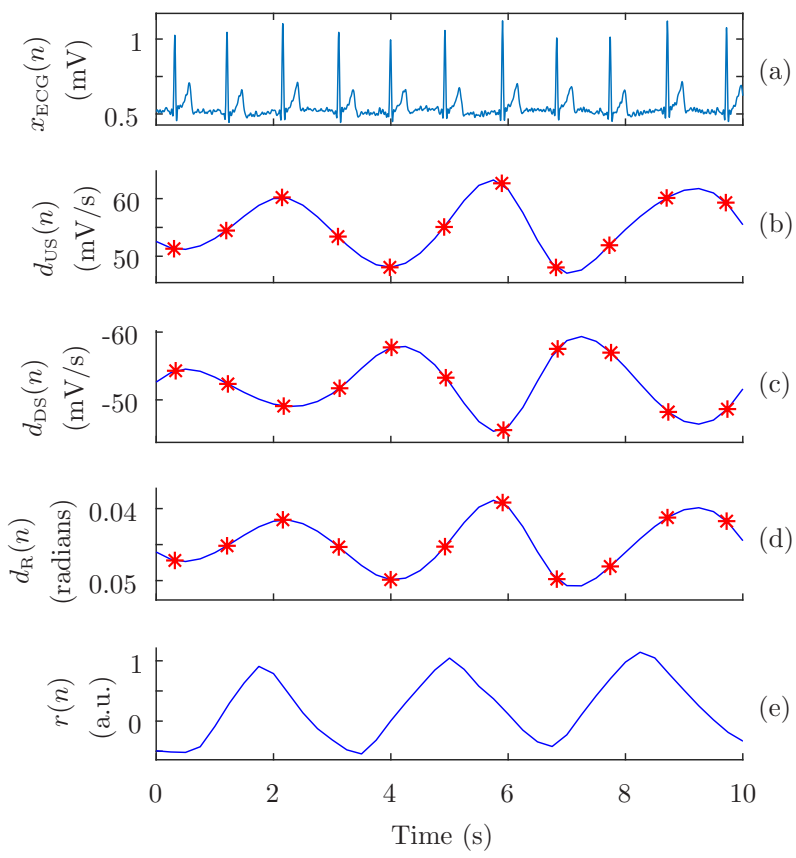

Figure 2: Example of recorded signals from a subject breathing at $0.3 \mathrm{~Hz}$, and the corresponding obtained series: ECG signal recorded by the wearable armband (a); Upwards to the R wave slope series (b); Downwards from the $\mathrm{R}$ wave slope series (c); R-wave angle series (d); and respiration recorded by Respibands (e). Red asterisks show the bet-to-beat measures of $\mathcal{I}_{\mathrm{Us}_{i}}, \mathcal{I}_{\mathrm{DS}_{i}}$, and $\phi_{i}$.

obtained by averaging only those $S_{j, k}(f)$ whose peakness fulfil 2 threshold-based criteria. The first is a fixed threshold (only peaked spectra take part in the average), and the second is a time-varying threshold which depends on the maximum peakness reached by all spectra of each time instant (only the most peaked spectra at each time instant take part in the average). The respiratory rate $\hat{f}(k)$ is estimated as the location of a peak in $\bar{S}_{\mathrm{k}}(f)$ whose choice is based on its amplitude and its proximity to $f_{\mathrm{R}}(k-1)$. No respiratory rate estimation is performed in those instants when no spectrum fulfils the peakness criteria. Further details are given in [6].

Respiratory rate was estimated from each one of the 3 EDR signals and from the combination of all the 3. Furthermore, respiratory rate was estimated by using the same algorithm from $r(n)$ and used as ground truth for evaluating the performance of the EDR methods.

\subsection{Performance measurements}

In order to evaluate the performance of the proposed method, the relative error of the obtained estimates with respect to those obtained from $r(n)$ was computed. Subsequently, the median and the interquartile range (IQR) of this relative error was computed for each examined respiratory rate. In addition, the percentage of estimates that obtained a relative error lower or equal to $3 \%\left(R_{e \leq 3}\right)$ was also computed.

\section{Results}

Respiratory rate was estimated $100 \%$ of the time in every case. Table 1 shows median, IQR, and $R_{e_{\mathrm{R}} \leq 3}$ of the relative error of the estimated respiratory rate, using the respiratory rate estimated from $r(n)$ as the gold standard.

Table 1: Obtained median (Med), IQR, and $R_{e_{\mathrm{R}} \leq 3}$ of relative error for each requested respiratory rate. Comb refers to the combination of the 3 studied EDR parameters.

\begin{tabular}{|c|c|c|c|c|c|}
\cline { 2 - 6 } \multicolumn{1}{c|}{} & Rate & $0.1 \mathrm{~Hz}$ & $0.2 \mathrm{~Hz}$ & $0.3 \mathrm{~Hz}$ & $0.4 \mathrm{~Hz}$ \\
\hline \multirow{3}{*}{$\mathcal{I}_{\text {US }}$} & Med (\%) & 0.90 & -0.11 & -0.21 & 0.02 \\
& IQR (\%) & 3.62 & 0.69 & 0.82 & 3.42 \\
& $R_{e \leq 3}(\%)$ & 59.68 & 100 & 93.75 & 67.69 \\
\hline \multirow{4}{*}{$\mathcal{I}_{\text {DS }}$} & Med (\%) & 0.47 & -0.24 & -0.10 & -0.09 \\
& IQR (\%) & 3.56 & 0.54 & 0.34 & 1.60 \\
& $R_{e \leq 3}(\%)$ & 67.64 & 100 & 100 & 78.46 \\
\hline \multirow{4}{*}{$\phi$} & Med (\%) & 0.67 & -0.28 & 0.18 & -0.11 \\
& IQR (\%) & 3.32 & 0.54 & 1.10 & 1.51 \\
& $R_{e \leq 3}(\%)$ & 69.35 & 100 & 100 & 81.54 \\
\hline \multirow{2}{*}{ है } & Med (\%) & 0.85 & -0.19 & -0.07 & -0.18 \\
ठे & IQR (\%) & 2.31 & 0.69 & 0.35 & 1.80 \\
& $R_{e \leq 3}(\%)$ & 75.81 & 100 & 100 & 81.54 \\
\hline
\end{tabular}

\section{Discussion}

A pilot study on respiratory information obtained from ECG signals recorded by a wearable armband for longterm monitoring has been presented. The algorithmic method was previously published and evaluated with 12standard-leads ECG in [6]. However, to the best of our knowledge, this is the first time that it is used with ECG recordings from a wearable device.

The wearable device is an armband developed in our lab at University of Connecticut and it includes a pair of dry electrodes which can record ECG signals from the arm. The dry electrodes and the locations of the electrodes in the arm is neither the optimal option for obtaining the best ECG signals nor for exploiting the influence of respiration over the ECG. However, it is more viable option than the conventional setup based on wet electrodes over the chest. The use of dry electrodes allows the long-term monitoring because they do not cause skin irritation while the conventional wet electrodes do, and placing the electrodes over the arm is more convenient and practical. Nevertheless, this represents a more challenging scenario than using wet 
electrodes over the chest because the signal-to-noise ratio is lower with dry electrodes. Moreover, the respirationsynchronous movement of the electrodes with respect to the heart is reduced when electrodes are placed over the arm. In this way, the respiration-related modulation in the ECG morphology may rely mainly on the impedance changes in the chest due to the airflow exchange.

Three QRS-morphology-based EDR parameters were analyzed: $\mathcal{I}_{\mathrm{US}}, \mathcal{I}_{\mathrm{DS}}$, and $\phi$. In addition, the information of these 3 EDR parameters was combined in order to examine if a more robust estimation can be obtained.

Obtained medians and IQRs of relative error (lower is better) were lower than $4 \%$ in every case. The 3 EDR signals reached a $R_{e \leq 3}$ (higher is better) of $100 \%$ when the examined respiratory rate was $0.2 \mathrm{~Hz}$. Moreover, $\mathcal{I}_{\mathrm{DS}}$ and $\phi$ reached a $R_{e \leq 3}$ of $100 \%$ also for a respiratory rate of 0.3 Hz. However, $\bar{\phi}$ obtained a higher or equal $R_{e \leq 3}$ than the other studied EDR signals for every examined respiratory rate. These results suggest that $\phi$ is the best choice among the 3 studied EDR signals if only one EDR signal is used.

The respiratory rate estimated from the combination of the 3 studied EDR signals obtained the same $R_{e \leq 3}$ for 0.2 , 0.3 , and $0.4 \mathrm{~Hz}$ than $\phi(100 \%, 100 \%$, and $81.54 \%$, respectively) and a higher $R_{e \leq 3}$ for $0.1 \mathrm{~Hz}(75.81 \%)$, suggesting that combining information from different EDR signals leads to more accurate results in some cases. It is worthy to note that in [6] the combination included information from different leads, and that the improvement obtained when combining EDR signals was more visible in time offering respiratory rate estimates than in estimation accuracy. In this work, every EDR signal and their combination offered respiratory rate estimates the $100 \%$ of the time. This may be attributed to the fact that the analyzed data were recorded from subjects while sitting quiet. A decrease in measuring time is expected while subjects are moving, and combination may offer a higher measuring time in those situations.

\section{Conclusions}

Respiratory rate can be obtained from ECG signals recorded by a wearable armband device, using a pair of dry electrodes over the arm. This remains a much more convenient setup for wearable devices than conventional wet electrodes over the chest, but also a more challenging scenario. However, medians and IQRs of relative error for normal ranges of spontaneous respiratory rate $(0.1-0.4 \mathrm{~Hz})$ were lower than $5 \%$. These results allow us to consider a wearable cardiorespiratory monitor based on an armband that records ECG, which could have a wide range of applications including sleep studies and chronic cardio and/or respiratory diseases daily monitoring. However, this is a pilot study which includes only 5 subjects. Further studies must be conducted in order to assess the performance of the methods with a higher number of subjects, and in different environments including while subjects are moving.

\section{Acknowledgements}

This project has received funding from the European Unions Framework Programme for Research and Innovation Horizon 2020 (2014-2020) under the Marie Skodowska-Curie Grant Agreement No. 745755. This work was supported also by Government of Aragón and European Social Fund (EU) through BSICoS group (T96), and by CIBER in Bioengineering, Biomaterials \& Nanomedicine (CIBER-BBN) through Instituto de Salud Carlos III. The computation was performed by the ICTS NANBIOSIS, specifically by the High Performance Computing Unit of CIBER-BBN at University of Zaragoza.

\section{References}

[1] de Chazal P, Heneghan C, Sheridan E, Reilly R, Nolan P, O'Malley M. Automated processing of the single-lead electrocardiogram for the detection of obstructive sleep apnoea. IEEE Trans Biomed Eng 2003;50:686-696.

[2] Varon C, Jansen K, Lagae L, Van Huffel S. Can ECG monitoring identify seizures? J Electrocardiol 2015;48:10691074.

[3] Hernando A, Lázaro J, Gil E, Arza A, Garzón JM, LópezAntón R, de la Cámara C, Laguna P, Aguiló J, Bailón R. Inclusion of respiratory frequency information in heart rate variability analysis for stress assessment. IEEE J Biomed Health Inform 2016;20:1016-1025.

[4] Frey U, Suki B. Complexity of chronic asthma and chronic obstructive pulmonary disease: implications for risk assessment, and disease progression and control. Lancet 2008; 372:1088-1099.

[5] Reyes BA, Posada-Quintero HP, Bales JR, Clement AL, Pins GD, Swiston A, Riistama J, Florian JP, Shykoff B, Qin M, Chon KH. Novel electrodes for underwater ECG monitoring. IEEE Trans Biomed Eng 2014;61:18631876.

[6] Lázaro J, Alcaine A, Romero D, Gil E, Laguna P, Pueyo E, Bailón R. Electrocardiogram derived respiratory rate from QRS slopes and R-wave angle. Ann Biomed Eng 2014; 42:2072-2083.

[7] Martínez JP, Almeida R, Olmos S, Rocha AP, Laguna P. A wavelet-based ECG delineator: Evaluation on standard databases. IEEE Trans Biomed Eng 2004;51:570-581.

[8] Mateo J, Laguna P. Analysis of heart rate variability in presence of ectopic beats using the heart timing signal. IEEE Trans Biomed Eng 2003;50:334-343.

Address for correspondence:

Jesús Lázaro

Department of Biomedical Engineering, UCONN

260 Gleenbrook Rd, Unit 3247, 06269 Storrs CT, USA

jesus.lazaro@uconn.edu 